Questions vives

\section{Questions Vives}

Recherches en éducation

$\mathrm{N}^{\circ} 33 \mid 2020$

Visées heuristiques, praxéologiques et critiques dans les recherches participatives en éducation

\title{
Expériences d'adolescents malades chronique et de suivis par des professionnels en santé mentale : réflexion sur les enjeux et visées d'une recherche?
}

Experiences of adolescents with chronic illness and follow-up by mental health professionnals: thinking about research challenges and aims?

\section{Martine Janner Raimondi}

\section{(2) OpenEdition}

Journals

\section{Édition électronique}

URL : http://journals.openedition.org/questionsvives/4624

DOI : $10.4000 / q u e s t i o n s v i v e s .4624$

ISSN : 1775-433X

Éditeur

Université Aix-Marseille (AMU)

\section{Édition imprimée}

Date de publication : 15 décembre 2020

ISBN : 978-2-912643-57-5

ISSN : 1635-4079

\section{Référence électronique}

Martine Janner Raimondi, «Expériences d'adolescents malades chronique et de suivis par des professionnels en santé mentale : réflexion sur les enjeux et visées d'une recherche? », Questions Vives [En ligne], № 33 | 2020, mis en ligne le, consulté le 25 mars 2021. URL : http://

journals.openedition.org/questionsvives/4624; DOI : https://doi.org/10.4000/questionsvives.4624

Ce document a été généré automatiquement le 25 mars 2021.

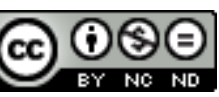

Questions Vives est mis à disposition selon les termes de la licence Creative Commons Attribution -

Pas d'Utilisation Commerciale - Pas de Modification 4.0 International. 


\section{Expériences d'adolescents malades chronique et de suivis par des professionnels en santé mentale : réflexion sur les enjeux et visées d'une recherche?}

Experiences of adolescents with chronic illness and follow-up by mental health professionnals: thinking about research challenges and aims?

\section{Martine Janner Raimondi}

1 Les recherches en Sciences de l'Éducation et de la Formation (SDEF) ont pour but de produire de nouvelles connaissances dans les différents champs de l'éducation/ formation relativement aux questions de société contribuant ainsi, à travers l'analyse de ses résultats à différentes formes de préconisation concernant les pratiques éducatives, comme l'a souligné l'HCERES (2018). Ceci nous invite à nous pencher sur les enjeux des recherches conduites. Si leurs dimensions heuristiques de production de savoirs et praxéologiques de transformation des pratiques apparaissent premières, n'avons-nous pas à interroger quels seraient les autres enjeux, notamment critiques, en repérant à quels niveaux précis ils interviennent alors même que leur portée est difficile à saisir avec exactitude. $Y$ aurait-il, en outre, un enchaînement repérable entre la visée heuristique et celle praxéologique, où intervient celle critique et en existe-t-il d'autres, lesquelles et à quel(s) niveau(x) ?

2 Pour répondre à ces questions qui incluent celle de l'articulation des enjeux d'une recherche, notre enquête s'appuiera sur une recherche pluridisciplinaire à laquelle nous avons largement contribué durant quatre années, qui nous permettra d'identifier combien la réflexion sur les visées ne saurait se défaire de la prise en compte des enjeux d'une recherche. 
Cette recherche ${ }^{1}$ a d'abord consisté à former une équipe pluridisciplinaire composée de praticiens hospitaliers, d'enseignants chercheurs en philosophie et en SEDF et de doctorants en philosophie autour de la problématique de la chronicité des pathologies chez les adolescents dont l'objectif visait à croiser les points de vue des soignants et des adolescents malades chroniques qu'ils suivent afin d'obtenir une vision qualitative fine du vécu de la maladie chronique à l'adolescence et de ses problématiques spécifiques. D'emblée, les visées heuristiques entendues comme perspectives de découverte étaient identifiées. Il s'agissait d'une part, de travailler à rendre compte du vécu de la maladie chronique appréhendé à partir des points de vue des patients adolescents et des soignants, qui les accompagnent; d'autre part, de recueillir des données empiriques expérientielles et les analyser, de façon à obtenir une vision qualitative de la maladie chronique adolescente vécue en propre ou soignée. Enfin, il s'agissait de croiser ces données entre elles afin d'appréhender plus précisément les expériences vécues de la maladie avec les concepts mobilisés dans le champ du soin. Dans le cadre de cet article, nous ciblerons les données recueillies en santé mentale.

4 Toute recherche nécessite que soit rendu nécessaire l'objet de son questionnement sous peine d'invalidation. Cette recherche résulte des constats de non observance encore appelée non compliance, comprise comme non prise en compte de la prescription médicale par le patient, en particulier en période d'adolescence. Les enjeux de ce manque d'observance peuvent entraîner des risques de morbidité - relatifs au degré de la maladie - ainsi que des risques de mortalité, tous deux considérés à l'aune des dépenses de santé. Au départ, cette étude s'inscrit dans un contexte de problématique de santé publique dans laquelle interfèrent des préoccupations non seulement financières mais également éthiques et politiques.

\section{Au départ une question de vie qui engage un fondement éthique et politique}

Levine \& Jacquin, (2008) précisent qu'à l'adolescence « la phase de non-observance est un passage quasi obligé, correspondant à l'expérimentation et à la découverte de soi » (Ibid., p.90). Ceci permet de comprendre pourquoi il est délicat d'atteindre un haut degré de compliance durant cette période. Pour autant, au-delà des écarts d'adéquation entre une prescription et son suivi par le patient, fût-il adolescent, n'y aurait-il pas à considérer ce qui se joue au niveau des sujets, malades au regard de la prescription donnée? Dit autrement, peut-être convient-il de dépasser la caractéristique développementale de l'adolescence posée comme invariant pour appréhender le sujet, son vécu expérientiel, ses éprouvés, ses ressentis, ses projets et ses désirs corrélativement à la prescription ainsi que la façon dont cette prescription lui a été donnée. À y regarder de plus près, qu'est-ce qui anime le chercheur en SDEF, qui précisément n'est pas thérapeute, à s'investir dans une recherche de ce type ?

6 La finalité d'apprentissage inhérente aux SDEF n'invite-t-elle pas à considérer le problème de la non observance des jeunes malades chroniques à l'aune des sujets et des relations qu'ils entretiennent avec leur maladie, leurs proches, leurs soignants précisément pour tâcher de repérer ce qu'ils apprennent de leurs expériences et de leurs relations? Ainsi par-delà la problématique de santé publique inhérente à un contexte socio-économique et historique, le point d'ancrage premier du chercheur en SDEF n'engage-t-il pas ipso facto une posture éthique qui considère le sujet 
en tant que sujet apprenant pris dans un maillage de relations entre la maladie, le thérapeutique et son environnement?

7 L'éthique déborde les contenus promus en matière de pratiques et de politiques en les interpellant soit parce que les réponses apportées jusque-là au « que dois-je faire? » ne fonctionnent plus; soit parce que les pratiques mises en œuvre entrent en contradiction avec quelque chose d'ordre supérieur, susceptible de provoquer l'indignation et poussant à se mobiliser, à agir, y compris en recherche. Trois voies principales, auxquelles correspondent autant de théories et de méthodes, s'offrent à lui, comme le précise Billier (2010) :

- Le conséquentialisme, qui s'intéresse fondamentalement aux conséquences des actes ou de leurs règles au regard d'un bien défini préalablement ;

- Le déontologisme, qui affirme qu'une action est morale si elle obéit aux principes absolus qui s'appliquent quelles qu'en soient les conséquences ;

-L'éthique des vertus, enfin, pour laquelle ce qui compte c'est le perfectionnement vertueux de l'agent moral :

Inévitablement, cette considération éthique entre en jeu dès le départ précisément parce que selon par où le chercheur entre, il n'effectuera ensuite pas le même chemin de recherche. Si nous suivons le procédé heuristique de Sidgwick (1874), l'éthique s'inscrit dans le sillage soit de la notion de «bien»; soit dans celui du «juste». Les conceptions du «bien» développées dès l'Antiquité grecque posent un idéal attractif dont la connaissance contribue au fait que le sujet désirerait contribuer à sa réalisation. Les conceptions du " juste » définissent un idéal moral impératif affirmant l'obligation pour le sujet d'y obéir par-delà ses souhaits. Dès lors, le sens de la valeur suprême, cardinale du respect de la personne humaine (Canto-Sperber, 2008) s'y trouve décliné différemment.

De quelle(s) éthique(s), le chercheur en SDEF se réclame-t-il ? le choix conséquentialiste aurait conduit le chercheur à une posture d'évaluation considérant essentiellement les conséquences de la non observance comme des actes posés par le sujet dont il conviendrait alors de repérer les effets positifs et négatifs.

Le choix d'une posture en considération de l'impératif catégorique du respect de personne humaine aurait placé le chercheur en posture de juge des activités de soin et d'auto-soin tant du côté des soignants que des malades.

11 Celui de l'éthique des vertus prend en compte une vulnérabilité humaine qui fait écho à l'éthique du Care telle que la développent Tronto (2009) et Kittay (2015). Il s'agit ici de dépasser les arguments abstraits, voire formels, pour prendre en compte l'effectivité des droits des personnes telles qu'elles sont dans la réalité. Or, la prise en compte de la fragilité et l'interdépendance entre les êtres humains ne sauraient oublier l'ensemble des êtres vivants et plus largement l'éco-système dans lequel ils vivent. L'éthique du Care, qui renvoie de fait au sujet relationnel et " attaché ", se construisant dans et par les relations qu'il tisse avec les autres et son environnement, ne saurait par conséquent éviter une considération critique des enjeux politiques au sens large. De fait, l'éthique du Care est née d'une critique des politiques prônées, comme le souligne Tronto (2009).

Un ordre politique qui présume que seules l'indépendance et l'autonomie constituent la nature de la vie humaine passe à côté de d'une bonne part de l'expérience humaine, et doit par ailleurs, d'une manière ou d'une autre, dissimuler cette question. Cet ordre doit par exemple séparer de manière rigide la vie publique de la vie privée : (Ibid., p. 182). 
Dans ce cadre, le chercheur en SDEF prendrait acte de la faillibilité/vulnérabilité propre au genre humain pour examiner ce que chacun des acteurs considérés a à signifier au chercheur sur ce qu'il vit dans son rapport à la maladie, ce qu'il en a appris de l'intérieur afin de donner à entendre leurs voix en vue d'une meilleure intercompréhension. L'enjeu de ces prises en considération respectives et croisées vise une compréhension des activités de soin en vue d'une éventuelle perfectibilité du soin ainsi qu'une compréhension des expériences vécues de la maladie chronique à l'adolescence. En effet, mieux connaître les vécus d'une maladie chronique permet d'une part, aux soignants de mieux comprendre les patients qui en sont porteurs et, d'autre part, permet aux patients eux-mêmes de mieux comprendre ce qui leur arrive, y compris en termes de soin. De ces considérations, il s'ensuit que l'enjeu de la posture éthique adoptée par le chercheur en SDEF comporte autant une visée praxéologique de soin d'autrui et de soi, qu'une visée d'apprentissage corrélative aux acquis d'expériences vécues de la maladie, pouvant conduire à une émancipation.

Sans aller jusqu'à une visée d'émancipation, le choix que j'ai fait durant la réalisation des enquêtes de terrain de cette recherche s'inscrit dans cette troisième voie.

\section{Une question de vie qui engage un fondement épistémologique en termes de conception du vivant}

14 Toute recherche pose au démarrage des ancrages théoriques auxquels s'ajoute une revue de l'art sur une question donnée. Autrement dit, en amont même de la revue de littérature sur un concept, il y a un socle sur lequel le chercheur fonde sa réflexion. Celui-ci n'est pas toujours d'emblée explicité. Or, c'est là que se situe la première pierre du raisonnement; il s'agit ici du premier enjeu épistémologique : celui qui fonde la connaissance. Dans cette recherche, au fondement est une conception du vivant en référence à Canguilhem (1966) :

Nous ne faisons pas profession [...] d'indéterminisme. Nous soutenons que la vie d'un vivant, fût-ce d'une amibe, ne reconnaît les catégories de vivant et de maladie que sur le plan de l'expérience, qui est d'abord épreuve au sens affectif du terme, et non sur le plan de la science. La science explique l'expérience mais ne l'annule pas pour autant: (Ibid., p. 131).

Par-delà les normes médicales largement diffusées dans nos sociétés connectées définissant les seuils de normalité pouvant être interprétés de façon fixiste et rigide alors même que la réflexion critique de Canguilhem nous apprend à les relativiser - il s'agit de faire place aux Sujets pour rendre possible et partant, compréhensible, une expérience de la maladie dans sa singularité. Cette dernière est reliée à la constitution du sujet qui la vit, au moment où il la vit in situ, c'est à-dire corrélativement aux relations établies avec son environnement physique, social et humain sur un territoire donné. Sans cet ancrage fondamental, cette recherche n'aurait eu aucun sens, c'est-àdire que les objectifs visés n'auraient pas été viables. C'est pourquoi nous pouvons affirmer que dans le cadre de cette recherche, dont l'objet même du questionnement résultait au départ du constat d'un manque de connaissances sur la maladie chronique adolescente en lien avec les constats de soignants sur la non-observance des jeunes, l'enjeu heuristique de départ s'appuie en réalité sur une réflexion critique ouvrant de nouvelles perspectives de recherche. 
16 Une telle «philosophie de l'individualité vivante » comme la qualifie Le Blanc (1998) défend la valeur subjective de l'expérience vécue, susceptible d'être narrée, alors même que le processus d'objectivation de la maladie et de son évolution dans le cadre d'une approche médicale stricte en droite ligne de l'evidence based medicine ne peut que refouler ces dimensions.

17 Or, narrer une expérience conduit à s'interroger sur le processus d'élaboration progressive du sens de celle-ci. Dès lors, Ricœur $(1983,1985)$ permet d'appréhender combien la vie éprouvée nécessite de passer par le récit associé au vécu permettant de faire comprendre en faisant "revivre» certains moments dans le cadre d'un développement réflexif de prise de conscience du sens déjà contenu dans le vécu initial. Pachoud (2017) précise toute l'importance de l'élaboration narrative en soulignant combien ce vécu :

[...] s'enrichit avec l'élaboration narrative, destinées à influencer des vécus ultérieurs. Autrement dit, il existe entre la vie et son élaboration, narrative un rapport de circularité permanente [...] en quoi consistent la réflexivité, le développement de la conscience et finalement l'expérience véritablement humaine : (Ibid., p. 70).

Une telle élaboration narrative est susceptible, pour le narrateur de «changer son agir» (Ricœur, 1990, p. 86) précisément parce que l'intelligibilité du sens de l'expérience vécue est de l'ordre du récit.

\section{Une question de vie qui engage une posture d'enquête en phénoménologie pragmatique}

S'attacher à appréhender ce qui se joue au cœur même des vécus et des éprouvés s'inscrit dans le sillage d'une approche phénoménologique en écho à Husserl et Ricœur. Comme l'a précisé Ricœur (1986) : «la phénoménologie parie pour cette discursivité primordiale de tout vécu»: (p.63). Plus encore, il s'agit d'en faire une lecture pragmatique telle que la propose Depraz (2009) afin d'accueillir ce qui se passe, y être attentif et ouvert en étant également attentif aux résonnances en soi (Depraz, 2014 ; Janner-Raimondi, 2017).

20 Il s'est agi d'appréhender combien le monde objectif renvoie en réalité à un monde intersubjectif dans lequel non seulement l'unité du corps vécu du je est capable de se déployer en faisant retour sur ce qui le constitue mais dans lequel la compréhension d'autrui passe par des résonnances avec mes propres kinesthèses. Dès lors, le choix méthodologique qui a suivi, a consisté à construire un protocole d'entretien double réalisé par un binôme de chercheurs.

21 Un premier entretien existentiel (Blanchet \& Gotman, 1992/2007) permet de recueillir des savoirs expérientiels, des perceptions, des éprouvés et des ressentis associés à la maladie pour les adolescents à l'accompagnement et/ou au suivi thérapeutique pour les professionnels de santé. Le récit est tout particulièrement requis dans ce premier entretien existentiel permettant ainsi de solliciter un récit sur le vécu et les expériences de la maladie d'où surgissent des événements pour les adolescents malades chroniques et leur(s) soignant(s). Ces événements ne sont pas considérés a priori indépendamment des narrateurs. Bien plutôt, ils émergent du récit lui-même via la mise en intrigue élaborée par les narrateurs. Il est possible que le choix des événements du narrateur soit différent selon le(s) narrataire(s) mais nous n'avons pas cherché à 
explorer cette piste, préférant appréhender ce que les narrateurs ont à signifier à propos des expériences vécues et des apprentissages qu'ils font de leur maladie à un instant « $t$ », indépendamment de sa variabilité.

Nous rejoignons là Ricœur pour lequel, il n'existe pas d'événement en dehors du travail de configuration/reconfiguration narrative qu'en fait le sujet. Une telle élaboration peut apparaître fortuite, c'est-à-dire qu'elle n'est pas nécessairement conscientisée par le sujet qui raconte son récit. De fait, il est écouté par le binôme de chercheurs et à ce niveau les chercheurs eux-mêmes peuvent renvoyer des éléments qu'ils ont perçus comme étant des événements.

Après ce premier entretien existentiel, un second est proposé sur la base d'une explicitation d'un moment laissé au choix du narrateur en lien avec l'un des deux ou trois événements repérés par les chercheurs. Il s'agit alors d'un entretien d'explicitation micro-phénoménologique (Vermersch, 1994/2014; Petitmengin, 2009), non-directif dans son contenu mais directif dans sa structure afin de décrire le vécu d'un événement présenté par l'interviewé en lien avec la maladie: moment du diagnostic, de la première hospitalisation, de ré-hospitalisation...

Chacun des deux chercheurs s'est spécialisé dans l'un des deux types d'entretien tout en s'étant acculturé à l'autre. C'est à l'issue du premier entretien que les deux chercheurs échangent brièvement sur les événements qui, de leurs points de vue, ont surgi du récit en laissant ensuite le choix à l'interlocuteur de porter plus spécifiquement son attention sur l'un ou l'autre des moments proposés (Janner Raimondi \& Depraz, 2018).

Sans rentrer dans les difficultés rencontrées pour obtenir les accords concernant les entretiens avec les jeunes, notamment relativement au Comité de Protection des personnes (CPP), nous disposons à ce jour, de 7 entretiens doubles auprès de 7 professionnels du soin composés de 3 psychiatres, 1 pédiatre, 1 infirmière et 2 psychologues auxquels s'ajoute 1 entretien double réalisé avec une jeune fille suivie par l'un des psychiatres entendus, 4 années plus tôt.

Une fois les entretiens retranscrits et les premières analyses thématiques de contenus faites concernant le(s) acquis d'expérience : autour de la maladie, du/des rapport(s) aux proches, du/des soin(s) et du/des suivi(s), nous avons procédé à des mutualisations entre chercheurs ainsi qu'à l'envoi des retranscriptions, voire des premières pistes d'analyse thématique.

\section{De quelques résultats et de leurs enjeux}

27 Au niveau méthodologique, nous repérons que le double entretien centré sur le suivi d'un jeune en particulier et non sur le suivi des jeunes en général comporte effectivement des éléments ciblés qui fournissent matière à explorer des événements précis liés au soin du jeune, propre à appréhender le vécu singulier. Le propos est moins général, il donne ainsi corps à l'expérience. Chez le patient, là aussi, les éléments narrés apportent des précisions qui peuvent être explorées.

28 L'ordre de réalisation du double entretien - récit puis explicitation - permet d'effectuer un recueil d'événements narrés propres à pouvoir alimenter un guidage vers des explicitations ultérieures. La narration est opérante à ce niveau. 

suivie, alors qu'elle était hospitalisée et se rappelle plusieurs événements comme le fait que certains soignants la forçaient à manger en lui tenant ses mains dans le dos; la suppression de son téléphone portable et des visites qui ont déclenché de son point de vue une radicale prise de conscience pour décider de s'en sortir ; ainsi que l'importance de la présence des pairs et des activités proposées notamment celles de type artistique (dessiner, peindre...).

Avant, j'avais le droit à mon téléphone et les visites. Et quand on m'a coupé ça... Parce que... enfin le fait que j'aie eu le droit à ça et puis qu'après on m'a coupé, je me suis rendu compte que c'était invivable en fait. Et c'est là où je... où j'ai compris que je devais vraiment m'en sortir...

Euh c'est... en fait ils ont... Bah ils n'arrivaient pas à voir comment j'allais m'en sortir, donc ils ont fait une rencontre avec les médecins de pédiatrie et les médecins de pédopsychiatrie et le Docteur... Et là ils ont fait une réunion pour se mettre d'accord et ils ont décidé d'augmenter ma sonde... enfin ils ont tout remis... d'enlever mon téléphone, d'enlever les visites et de me les autoriser petit à petit que je reprenais du poids : (JF. $16^{\circ} \mathrm{mn}$ de l'entretien existentiel).

Du côté du médecin psychiatre, il est indiqué combien il fallait démêler en début de prise en charge les nombreux symptômes successivement développés par la jeune fille (phobie scolaire, asthme, problèmes relationnels avec l'un des parents, puis problèmes 
cardiaques) pour identifier in fine la problématique centrale de l'anorexie. Différentes conceptions du soin s'étaient alors trouvées en tension, selon qu'il s'agisse du diagnostic pédiatrique (l'adolescente était alors âgée d'une quinzaine d'années) ou psychiatrique, auxquels se sont ajoutées les tensions concernant les modalités de traitement de l'anorexie, elle-même. Ces tensions et les interventions croisées de plusieurs thérapeutes de domaines de soin différents ont finalement conduit à opter pour des conditions de vie hospitalisée dans le secteur pédiatrique, puis pédopsychiatrique. Or, c'est au cours d'un séjour en pédopsychiatrique que la jeune femme a développé sa volonté de s'en sortir.

Les quelques éléments recueillis tendent à corroborer la pertinence de notre dispositif méthodologique d'enquête. Pour autant, cette pratique de recherche reste à poursuivre afin de disposer d'éléments en quantité suffisante pour se prononcer davantage.

Les résultats confirment l'écart entre ce que les anthropologues de la santé (Kleinman, 1998) nomment la maladie diagnostiquée et la maladie ressentie. Cet écart se révèle particulièrement saillant lorsque l'on croise les deux entretiens : le psychiatre qui parle du suivi de la jeune fille et cette dernière qui raconte son expérience de la maladie en institution.

Si la maladie diagnostiquée renvoie à une modification ou au dysfonctionnement des processus biologiques, psychologiques ou psychiques, la maladie ressentie incorpore l'expérience et la perception individuelles relativement aux difficultés issues de la maladie diagnostiquée ainsi qu'aux réactions sociales face à cette maladie, y compris au niveau des ressentis éprouvés.

La maladie ressentie n'est pas qu'individuelle, les voies qui la constituent sont plurielles et souvent conflictuelles. Cette référence fait sens avec le récit de la jeune femme qui se rappelle avoir demandé à sa mère de lui rapporter un livre expliquant ce qu'est l'anorexie. Dans cet ouvrage, l'une des activités proposées consistait à évaluer sa propre morphologie sur une échelle graduée sous forme d'images de morphologies plus ou moins corpulentes. Le croisement des appréciations sur la façon dont la jeune fille se voyait et celle de sa mère fut pour l'adolescente un autre moment de prise de conscience. Ainsi, comme le synthétise Almeida-Fliho (2006), le sens de la maladie regroupe un ensemble d'expériences, de mots, de sentiments et de pratiques sociales.

40 Mais, au-delà des problématiques de prise en compte des sujets dans leur individualité, il y a lieu de considérer l'impact d'un postulat d'universalité des troubles, qui écarte toute prise en compte des données sociales et culturelles alors même que les variables sociales et culturelles pourraient être associées à l'occurrence des maladies (Bibeau \& Corin, 1995).

\section{5. Éléments de conclusion}

41 Comme cela a été repéré, cette recherche comporte plusieurs enjeux épistémologiques articulés à des enjeux critiques permettant de préserver une visée d'ordre éthique et politique à fins praxéologiques de transformation des pratiques.

En premier, il s'est agi de développer une connaissance basée sur la parole des acteurs : non pas «parler sur» mais "parler avec»; non pas recueillir des données mais échanger, dialoguer en cherchant à comprendre les personnes premières concernées dans le cadre d'une relation intersubjective où il s'agit d'apprendre de lui et avec lui 
(car les entretiens peuvent favoriser la prise de conscience des personnes avec lesquelles l'entretien est réalisé) en s'assurant que les premiers éléments de compréhension élaborés par le(s) chercheur(s) soient soumis, lorsque cela était possible, aux interlocuteurs afin d'éviter le risque autant d'une surinterprétation que d'une incompréhension.

Outre cet enjeu, il s'agit comme le précisent Giroux \& Gzil (2012) de repérer combien interpréter un état de souffrance comme une maladie plutôt qu'en termes de possession ou d'épuisement, revient à lui appliquer un schème causal qui permet de l'expliquer, mais aussi d'en prévoir le étapes d'évolution afin de tâcher d'en réguler le cours, voire, d'en prendre le contrôle. Le biopouvoir invite ici le chercheur à se montrer vigilant et critique quant aux enjeux de domination via le/les soins prodigués. En effet, le schème explicatif rend épistémologiquement possible le travail de soin du médecin, via le traitement administré précisément parce que la maladie appréhendée comme concept entraîne à sa suite une démarche, posant un diagnostic, un pronostic et une thérapie. Or, la notion de santé est influencée par la question des valeurs qu'une société se donne en un temps et un lieu donné. En tant que cadre général de ce qui est estimé comme valant pour tous les membres d'une telle société, la santé se comprend alors comme 'idéal régulateur' (Giroux \& Gzil, 2012, p. 225) des usages de la maladie.

En fait, la centration de la médecine sur la maladie comme objet d'étude et d'investigation tend à évacuer non seulement la prise en compte du patient en tant qu'être humain vulnérable, mais également celle du patient qui apprend de sa maladie et sur sa maladie. Ici également, le chercheur est amené à adopter une posture critique en vue d'appréhender plus finement les enjeux socio-politiques et éthiques de sa recherche. Dès lors, favoriser une meilleure écoute du vécu de la maladie invite à redonner place au patient, offrant ainsi un contrepoids au biopouvoir de la médecine (Dominicé \& Jacquemet, 2009).

Les visées heuristiques de cette recherche ont notamment conduit à reprendre l'examen critique des notions de santé et de «normal » développé par Canguilhem (1966) dans sa dénonciation de la conception du normal chez Auguste Comte, dans la Quarantième leçon du Cours de Philosophie Positive (1864), fondée sur la biologie. En suivant Canguilhem, le normal ne se présente plus comme une unité théorique construite rationnellement qui s'impose comme unique référence. À la norme biologique de bon fonctionnement de l'organisme qui pointe l'état de santé sous forme statique, l'état de santé de chacun, aujourd'hui, s'apprécie davantage au regard de la fourchette statistique «admise comme normale». Pour autant, le «normal» comme moyenne chiffrée ne suffit pas à approcher la notion de santé, car il est sans rapport avec le vécu subjectif de la maladie, lui-même en lien avec les notions de territoires et de cultures.

Précisément, ce que nous apprend cette recherche c'est que la maladie chronique ne s'aurait s'appréhender de façon fixiste et définitive. Elle apparaît au contraire fluctuante avec des moments de crise et d'autres de répit alors même qu'elle s'inscrit durablement dans le corps. Elle apparaît aussi parfois aléatoire sans que le corps médical ni les patients eux-mêmes ne comprennent ce qui se passe. La maladie chronique, au fond, fait échec aussi bien à l'idée de norme biologique unique de référence, qu'aux soins compris comme traitement unique sous forme de protocoles prédéfinis. Les expériences vécues des suivis croisées avec les expériences vécues de la maladie chronique réinterrogent le sens même du mot rétablissement qui ne peut 
s'appréhender comme rétablissement complet de l'état antérieur, obligeant ainsi à redéfinir la notion même de santé, tant il s'agit en fait de vivre avec au mieux.

Le Blanc (2002) rappelle combien « la normalisation est une normativité instituée tandis que la normativité est une normalisation contestée. » (Ibid., p. 221). En cela, il nous invite à mieux appréhender la part et la marge des sujets pris dans leur singularité opérant des réorganisations au niveau des normes du vivant. Dès lors soigner et accompagner les malades chroniques ne sauraient éviter de solliciter ce travail de normativité/ autonormativité propre à chacun, $a$ fortiori pour des adolescents en âge d'interroger de façon critique les normes transmises. Il s'agirait alors de solliciter un tel travail pour éviter le risque d'une accentuation de la chronicisation de la maladie potentiellement liée à l'exigence d'un respect strict des préconisations médicales unilatérales pouvant conduire autant à leur rejet qu'à la perte de la personne malade. Nous voyons ici poindre l'articulation des précédents enjeux avec des visées praxéologiques définies autant dans l'intérêt des patients que des soignants.

Enfin, à la question de savoir ce qu'apporte le regard des SDEF dans un tel projet pluri disciplinaire, la réponse que je ferai, réside dans la préoccupation composée d'un triptyque articulant nécessairement les visées suivantes: éthique, heuristicoépistémologique et praxéologico-politique. Il s'agit en effet d'articuler la vigilance éthique de respect et de prise en compte avec tact des personnes sollicitées par la recherche, tout particulièrement lorsque se font jour la blessure et la force en lien avec la maladie (Barrier, 2009), quand se trame l'agir, le subir et le pâtir où se joue la compréhension fine de la souffrance (Marin, 2017), à la visée heuristique et épistémologique des apprentissages élaborés à partir de ces expériences vécues de la maladie et des soins prodigués. Mais, ces deux volets seraient vains sans la visée praxéologique, qui opère inévitablement au plan politique en contribuant à œuvrer à plus ou moins long terme au pouvoir d'agir des acteurs. Et s'il fallait considérer l'ordre des panneaux de ce triptyque, nous pourrions dire que l'éducatif suppose et engage l'éthique, l'heuristico-épistémologique puis le politique sous peine de n'être qu'un dressage arbitraire.

\section{BIBLIOGRAPHIE}

Almeida-Filho, N. de (2006). Modèles de santé et de la maladie... Rupture, revue transdisciplinaire de santé, vol. 11, n 1, p. 122-146.

Barrier, P. (2009). Education thérapeutique : un enjeu philosophique pour le patient et son médecin. Actualité et dossier en santé publique, $\mathrm{n}^{\circ} 66, \mathrm{p}$. 57-78.

Bibeau, G., Corin, E. (1995). Culturaliser l'épidémiologie psychiatrique : les systèmes de signes, de sens et d'actions en santé mentale, in F. Trudel, P. Charest, \& Y., Breton, éds. La construction de l'anthropologie québécoise. Éloges offerts à Marc-Adélard Tremblay. (pp. 105-148). Sainte-Foy : Les Presses de l'Université Laval. 
Billier, J-C. (2010). Introduction à l'éthique. Paris : PUF.

Blanchet, A. \& Gotman, A. (1992/2007). L'enquête et ses méthodes : l'entretien. Paris : Nathan.

Canguilhem, G. (1966). Le normal et le pathologique. Paris : PUF, $1^{\circ}$ éd. 1943.

Charest, P., Trudel, F. \& Breton, Y. (Dir. 1994). M. A. Tremblay ou la construction de l'anthropologie québécoise. Québec : Presses universitaires de l'université Laval.

Comte, A. (1864) Cours de philosophie positive. Tome 3, Quarantième leçon : Considérations philosophiques sur l'ensemble de la science biologique. (pp. 187-338). Paris : J-P. Baillères \& Fils. Depraz, N. (2009). Plus sur Husserl : une phénoménologie expérientielle. Paris : Atlande.

Depraz, N. (2014). Attention et vigilance à la croisée de la phénoménologie et des sciences cognitives. Paris : PUF.

Dominicé, P. \& Jacquemet, S. (2009). Formation et santé. Note de synthèse. Savoirs, 19, p. 9-38.

Giroux, E. \& Gzil, F. (2012). Les concepts de santé et de maladie. In : E. Giroux \& M. Lemoine (Dir.) Philosophie de la médecine. (pp. 223-258). Paris : Vrin.

Jacquin, P. \& Alvin, M. (2008). Difficultés d'observance dans les maladies chroniques à l'adolescence : comprendre pour agir. Archives pédiatriques 15/2008, 89-94.

Janner Raimondi, M. (2017). Visages de l'empathie en éducation et en formation. Nîmes : Champ Social. Janner Raimondi, M. \& Depraz, N. (2018). Approcher l'expérience vécue des suivis d'adolescents malades chroniques en santé mentale, Le Sujet dans la Cité, n 9, 75-92.

Janner Raimondi, M., Bedoin, D. \& Baeza, C. (2019). Configurations d'espace d'alliance thérapeutique et de négociation du soin entre un adolescent malade chronique, ses parents et des soignants. Phronesis, $\mathrm{n}^{\circ}$ 8, p. 60-71.

Janner Raimondi, M. (2019). Brosser le portrait d'adolescents malades chroniques : approche phénoménologique du vécu existentiel singulier de la maladie. In E. Théodoropoulou (Dir.). Philosophie en praxis : engagements politiques, éthiques et éducatifs. Actes Biennale de Philosophie Pratique, Rhodes p. 150-165.

Kleinman, A. (1998). The illness narratives: suffering healing and the human condition. New York: Basic books.

Kittay, E. F. (2015). Le désir de normalité. Quelle qualité de vie pour les personnes porteuses de handicap cognitif sévère? ALTER, European Journal of Disability Research, 9 (2015), p. 175-185.

Le Blanc, G. (2002). La vie humaine : anthropologie et biologie chez Georges Canguilhem. Paris : PUF.

Marin, C. (2017). Penser la souffrance avec Paul Ricœur. Philosophie, 2017/1, n 132, 121-130.

Noël, D. (2006). Pour une réponse transculturelle en matière de santé mentale autochtone. Santé mentale au Québec, 2006, XXXI, 2, 153-164.

Pachoud, B. (2017).

Petitmengin, C. (2014). De l'activité cérébrale à l'expérience vécue. In : J-F. Dortier (éd.). Le cerveau et la pensée. (pp. 59-69). Paris : Ed. des Sciences Humaines.

Pinsart, M-G. (éd. 2008) Narration et identité. De la Philosophie à la bioéthique. Paris : Vrin.

Ricœur, P. (1983-1984). Temps et récits. II, III. Paris : Seuil.

Ricœur, P. (1986). A l'école de la phénoménologie. Paris : Seuil. 
Saillant, F. (1999). Vers une anthropologie des soins, Soins, corps, altérité, Anthropologie et Sociétés, 23-2.

Sidgwick, H. (1874). The method of Ethics. Cité par J-C. Billier (2010). Ibid., p. 23-24.

Tronto J. (2009). Un monde vulnérable. Pour une politique du Care. Paris : La Découverte, trad.

Anglaise. Moral Boundaries. A political Argument for un Ethic of Care. New-Yok : Routledge, 1993.

Vermersch, P. (1994, 2014). L'entretien d'explicitation. Paris : ESF.

Vermersch, P. (2012). Explicitation et phénoménologie : vers une psychophénoménologie. Paris : PUF.

\section{NOTES}

1. Recherche Les adolescents face à la maladie chronique : à la croisée de la médecine, de la philosophie et des sciences de l'éducation, financée par le Grand Réseau de Recherche de Haute-Normandie (GRR). Porteur Pr. N. Depraz, Université Rouen, laboratoire Eriac.

\section{RÉSUMÉS}

Si les dimensions heuristiques de production de savoirs et praxéologiques de transformation des pratiques apparaissent premières dans les recherches conduites en sciences de l'éducation et de la formation, n'avons-nous pas à questionner s'il existe d'autres enjeux et visées, si oui lesquelles et quelle(s) seraient leur(s) articulation(s) ? Par exemple, à quels niveaux se situe l'enjeu critique d'une recherche? Au nom de quoi se fait-elle? Pour répondre à ces questions, notre réflexion méta s'appuiera sur une enquête de terrain, à laquelle nous avons largement contribué durant quatre années, concernant les expériences de la maladie chronique à l'adolescence et de leurs suivis thérapeutiques, notamment dans le champ de la santé mentale. Nous chercherons à identifier comment s'intriquent les visées et enjeux d'une recherche.

If the heuristic dimensions of knowledge production and praxeological transformation of practice appear primary in researche conducted in education and training sciences, do we not have to question if there are other challenges and targets, if yes wich ones and what would be their articulation(s)? For example, at what levels is the critical issue of a research? In the name of what is it done? To answer the questions, our meta analysis will be based on a field survey, to wich we have largely contributed for four years, concerning the experiences of chronic illness in adolescence and their therapeutic follow-ups, especially in the field of mental health. We will seek to identify how the aims and challenges of a research are knitted.

\section{INDEX}

Mots-clés : expérience, maladies chroniques, éthique, épistémologie, praxéologie

Keywords : experience, chronic diseases, ethic, epistemology, praxeology. 


\section{AUTEUR}

\section{MARTINE JANNER RAIMONDI}

Professeur des universités en sciences de l'éducation et de la Formation, université Sorbonne

Paris Nord, UR Experice 